\title{
GENERAL TREATMENT OF ALPHABET-MESSAGE SPACE AND INTEGRAL REPRESENTATION OF ENTROPY
}

\author{
By Hisaharu Umegaki
}

\section{Introduction.}

In this paper we shall clarify a topological structure of the alphabet-message space of the memory channel in information theory, and study the integral representation of entropy amount from a general view point of a certain generalized message space. In order to apply to the general theory of entropy, the present fashion will develop a message space into more general treatment, in which the basic space $X$ will be assumed to be totally disconnected. As will be shown in $\S 2$, the alphabet-message space $A^{I}$ is a totally disconnected compact space, and in $\S 3$, a kind of theorem relative to sufficiency for a $\sigma$-field generated by a partition and a homeoporphism (cf. Theorem 2) and the others (Theorems 3 and 4) are concerned with the semi-continuity of entropy amount which are general form of Breiman's Theorem [1]. Finally, in $\S 4$, it will be discussed about the function $h(x)$ found by Parthasarathy [7] whose integral defines the corresponding amount of entropy (cf. Theorem 5). It is also shown that the results in [9] can be generalized (cf. the footnote 2) below). The function $h(x)$ may give useful and interesting tool for the general theory of entropy of measure preserving automorphism or flow over a probabiltiy space.

\section{Structure of message space.}

A Hausdorff space $X$ is called totally disconnected if $X$ has a base consisting of closed-open sets, clopen say. In such a space $X$, a measure $\mu$ is called normal, if $\mu$ is regular and the mass of every non-dense set is zero. The space $X$ is called hyper-Stonean, if it is compact and the union of carriers of all finite normal measures is dense in $X$. Such a space $X$ is characterized by the existence of a normal measure $\mu$ (not necessarily finite) on $X$ such that $\mu(G)>0$ for every nonempty open set $G$ (cf. Dixmier [2]). Whence, the Banach space $C(X)$ of real continuous functions on $X$, with sup-norm $\|\cdot\|$, is isometrical and lattice isomorphic to the conjugate space of $L^{1}$-space $L^{1}(X, \mu)$. It is known that, these concepts on $X$ are closely related with the theories of Boolean algebras and especially of operator algebras (=von Neumann algebras, cf. Dixmier [3]).

Received September 4, 1963. 
In this section, for the sake of functional analysistic necessity and interest, it will be investigated on the topological structure of the space $A^{I}$, defined below.

Let $A$ be an alphabet, i.e., a set consisting of finite number of elements. Put $A_{k}=A(k=0, \pm 1, \pm 2, \cdots)$ and denote $A^{I}=\times_{k=-\infty}^{\infty} A_{k}$ the doubly infinite product set. $A^{I}$ will be called message space. In the memory channel, the input space of alphabet information source is taken as the measurable space $\left(A^{I}, \mathfrak{A}\right)$ where $\mathfrak{A}$ is the $\sigma$-field generated by all finite dimensional cylinder sets in $A^{I}$.

Since each coordinate space $A_{k}$ is a finite set, they are compact metric spaces relative to each discrete topology, hence by Tychonoff's theorem, $A^{I}$ is also compact with countable base relative to the weak product topology and is metrizable. For each point $a \in A^{I}=\left(\cdots, a_{-1}, a_{0}, a_{1}, a_{2}, \cdots\right)$, denote

$$
\left[a_{m}, \cdots, a_{n}\right] \quad(m \leqq n)
$$

the $(n-m+1)$-length message, say finite message, i.e., the set of all $\mathfrak{x} \in A^{I}$ whose $k$-th coordinate equals to $a_{k}(k=m, \cdots, n)$. The messages are obviously clopen. Let $U$ be any non-empty open set in $A^{I}$. Then there exists a finite set of integers $J \subset I$ such that the projections $\operatorname{pr}_{k}(U)$ of $U$ into $k$-th coordinate spaces $A_{k}, k \in I-J$, are the whole spaces $A_{k}$, respectively. Consequently, putting $m=\min \{k ; k \in J\}$ and $n$ $=\max \{k ; k \in J\}$, then for any fixed $a \in U,\left[a_{m}, \cdots, a_{n}\right]$ is contained in $U$. Thus we obtain the following

THEOREM 1. The message space $A^{I}$ is a compact metric and totally disconnected space relative to the product topology, in which the shift is a homeomorphism on $A^{I}$ and the $\sigma$-field $\mathfrak{A}$ consists of all Borel sets. Especially every finite message is a clopen set and the family of all finite messages is base in $A^{I}$ as its topology. Furthermore $A^{I}$ is not hyper-Stonean.

Since the shift is obviously continuous and one to one on $A^{I}$ onto itsef, it is homeomorphism on $A^{I}$. Hence the proof is remained only in the last part. If $A^{I}$ is hyper-Stonean, then $C\left(A^{I}\right)$ is identified with the conjugate space of $L^{1}\left(A^{I}, \mu\right)$ for certain normal measure $\mu$ on $A^{I}$. Let $\left\{E_{n}\right\}$ be an infinite sequence of mutually disjoint and non-empty clopen sets in $A^{I}$, and let $M$ be the weakly* clopen subspace of $C\left(A^{I}\right)$ generated by the sequence of the characteristic functions $\left\{C_{E_{n}}\right\}$, where the closure is concerning the weak topology as conjugate space. While $M$ is separable relative to the norm-topology, because so is $C\left(A^{I}\right)$. Therefore $M$ must be finite dimensional. This is a contradiction. Thus $A^{I}$ is not hyper-Stonean.

\section{Properties of entropy functional.}

In order to devolope the theory of entropy over the message space $A^{I}$ from a general point of view, we shall take as an information basic space a totally disconnected compact Hausdorff space $X$ with a fixed homeomorphism $S$, which contains the case of $A^{I}$ as a special case. Here, every colopen set in $X$ and the homeomor- 
phism $S$ are corresponding to the finite message and the shift in $A^{I}$, respectively.

Denote $\mathfrak{X}$ the $\sigma$-field of all Borel sets in $X$. Let $\boldsymbol{P}_{\mathrm{S}}$ be the set of all $S$-invariant regular probability measures $p, q, \cdots$ on $X$. Let $c^{2}$ be a fixed covering of $X$ consisting of clopen sets such as any pair $U, U^{\prime} \in \mathscr{Z}^{\prime}$ being disjoint. Such an $\mathscr{Z}$ is always finite by the compactness of $X$ and it will be called clopen partition of $X$. As in $\S 2$ of [9], putting $\mathscr{I}_{n}=\bigvee_{k=1}^{n} S^{-k} \mathscr{F}_{\mathbb{Z}}$ or $\mathscr{I}_{\infty}=\bigvee_{n=1}^{\infty} \mathscr{I}_{n}$ the $\sigma$-field generated by $\left\{S^{-k} \mathscr{I}_{k=1}^{n}\right.$ or $\left\{\mathscr{I}_{n}\right\}_{n=1}^{\infty}$, respectively. Then the entropy $H(p)=H(p, \mathbb{a}, S)$ of each $p \in \boldsymbol{P}_{\mathrm{S}}$ is defined by the limit

$$
H(p)=-\lim \frac{1}{n} \sum_{U} p(U) \log p(U) \quad(n \rightarrow \infty)
$$

where $\Sigma_{U}$ means the summation over $U$ of the atomic sets in $\mathscr{Z} \vee \mathscr{I}_{n-1}$.

For any $p \in \boldsymbol{P}_{\mathrm{S}}$, denote $P_{p}\left(U \mid \mathscr{Z}_{2}\right)$ and $P_{p}\left(U \mid \mathscr{F}_{\infty}\right)$ the conditional probability functions of $U \in \mathscr{Z}$ conditioned by $\mathscr{Z}_{n}$ and $\mathscr{Z}_{\infty}$ in the measure space $(X, \mathfrak{X}, p)$, respectively, where $\mathfrak{X}$ is the $\sigma$-field of all Borel sets, and for a pair $p, q \in \boldsymbol{P}_{\mathrm{S}}$, denote $q \ll p$, when $q$ is absolutely continuous with respect to $p$. Then we prove

THEOREM 2. For any pair $p, q \in \boldsymbol{P}_{\mathrm{S}}$ with $q \ll p$, it holds that

$$
P_{p}\left(U \mid \mathscr{F}_{\infty}\right)(x)=P_{q}\left(U \mid \mathscr{F}_{\infty}\right)(x) \quad \text { q-a.e. } x \in X \text { and for every } U \in \mathscr{F}_{\text {. }}
$$

Proof. Putting $\mathscr{B}$ the $\sigma$-subfield generated by $\mathscr{Z}$ and $S^{-k} \mathscr{H},(k= \pm 1, \pm 2, \cdots)$, then, since $(X, \mathscr{B}, p)$ is separable for each fixed $p \in \boldsymbol{P}_{\mathrm{S}}$, from the generic property of $\mathscr{B}$, it follows that every $S$-invariant set $B \in \mathscr{B}$ belongs to $\mathscr{F}_{\infty}(\bmod p)$. Besides for every $p \in \boldsymbol{P}_{S}$, putting $p^{\prime}=(p \mid \mathscr{B})$, the restriction of $p$ over $\mathscr{B}$, then $p^{\prime}$ is $S$-invariant probability measure over $(X, \mathscr{B})$ and $P_{p}\left(U \mid \mathscr{L}_{\infty}\right)=P_{p^{\prime}}\left(U \mid \mathscr{Z}_{\infty}\right)$ for every $U \in \mathscr{F}_{\text {on }} C_{p}$ the carrier of $p$ over $\mathscr{B}$. Since $C_{p}$ is $S$-invariant, it is $\mathscr{Z}_{\infty}$-measurable. Taking $p, q \in \boldsymbol{P}_{S}$, $q \ll p$, then $q^{\prime} \ll p^{\prime}$ and the Radon-Nikodym derivative $d q^{\prime} / d p^{\prime}$ is $S$-invariant and $\mathscr{B}$ measurable, and hence $\mathscr{F}_{\infty}$-measurable $(\bmod p)$. Therefore for every $V \in \mathscr{F}_{\infty}$ and every $U \in \mathscr{Z}$

$$
\begin{aligned}
\int_{V} P_{q}\left(U \mid \mathscr{F}_{\infty}\right)(x) d q(x) & =\int_{V} C_{U}(x) d q(x)=\int_{V} C_{U}(x) \frac{d q^{\prime}}{d p^{\prime}}(x) d p(x) \\
& =\int_{V} P_{p}\left(U \mid \mathscr{F}_{\infty}\right)(x) \frac{d q}{d p}(x) d p(x)=\int_{V} P_{p}\left(U \mid \mathscr{Z}_{\infty}\right)(x) d q(x)
\end{aligned}
$$

and (2) holds, where $C_{U}(x)$ is the characteristic function of $U$.

This theorem implies that $\mathscr{F}_{\infty}$ is a sufficient subfield for the set $\left\{p^{\prime} ; p \in \boldsymbol{P}_{S}\right\}$ of measures on $(X, \mathscr{B})$ (in the sense of Halmos-Savage).

Theorem 3. For each $p \in \boldsymbol{P}_{\mathrm{S}}$, there exists, uniquely within p-a.e., a bounded, upper-semicontinuous and $\mathscr{Z}_{\infty}$-measurable function $h_{p}(x)$ on $X$ such that

$$
h_{p}(x)=-\sum_{U \in \mathscr{E}} P_{p}\left(U \mid \mathscr{L}_{\infty}\right) \log P_{p}\left(U \mid \mathscr{Z}_{\infty}\right)(x) \quad \text { p-a.e. } x \in X .
$$

Proof. Since each $V \in \mathscr{L}_{n}$ is clopen, $P_{p}\left(U \mid \mathscr{F}_{n}\right) \in C(X)$. Putting

$$
h_{p, n}(x)=-\sum_{U \in \mathscr{F}} P_{p}\left(U \mid \mathscr{I}_{n}\right)(x) \log P_{p}\left(U \mid \mathscr{I}_{n}\right)(x),
$$


then $h_{p},{ }_{n} \in C(X)$ and the sequence $\left\{h_{p},{ }_{n}\right\}_{n=1}^{\infty}$ is monotone decreasing, by the Jensen's inequality, say

$$
h_{p}(x)=\lim _{n \rightarrow \infty} h_{p},{ }_{n}(x),
$$

and $h_{p}(x)$ is upper-semicontinuous on $X$. Furthermore, since each $h_{p},{ }_{n}(x)$ is $\mathscr{F}_{n}$ measurable, $h_{p}(x)$ is $\tilde{z}_{\infty}$-measurable. Besides, $\left\{h_{p}, n\right\}$ is semi-martingale over the probability space $(X, \mathfrak{X}, p)$, and hence by the semi-martingale convergence it satisfies (3).

By Theorem 3 and by the well known theorem of McMillan, it holds that

$$
H(p)=\int_{X} h_{p}(x) d p(x)
$$

and $h_{p}(x)$ is S-invariant in the a.e. sense.

THEOREM 4. The functional $H(p)$ over $\boldsymbol{P}_{\mathrm{S}}$ is weakly* upper-semicontinuous, where the continuity is one with respect to the weak topology as functional over the Banach space $C(X)$.

Proof. By the proof of Theorem 3, for every $p \in \boldsymbol{P}_{\mathrm{S}}$

$$
\int_{X} h_{p},{ }_{n}(x) d p(x)\left(=H_{n}(p), \text { say }\right) \downarrow \int_{X} h_{p}(x) d p(x)=H(p) \quad(n \rightarrow \infty)
$$

and hence it is sufficient to prove the weak* continuity of $H_{n}(p)$. But this follows immediately from that

$$
\begin{aligned}
H_{n}(p) & =-\sum_{U \in \nsubseteq} \int_{X} C_{U}(x) \log P_{p}\left(U \mid \mathcal{F}^{\prime} n\right)(x) d p(x) \\
& =\sum_{U \in \mathscr{Z}} \sum_{V \in \Phi_{n}}[p(U \cap V) \log p(V)-p(U \cap V) \log p(U \cap V)]
\end{aligned}
$$

and that every $U \in \mathcal{C}_{2}$ and $V \in \mathscr{C}_{n}$ are clopen where $\sum_{V \in \bigotimes_{n}}$ means the summation over atomic $V$ in $\mathscr{Z}_{n}$.

\section{Integral representation of amount of entropy by a universal function.}

We shall show the theorem of Parthasarathy [7] for the present case. Assume the notations given in $\S 3$.

THEOREM 5. For any clopen partition ci, there exists unversally a Borel measurable functions $h(x)=h(x, a, S)$ on $X$ such that it is bounded, non-negative, $S$ invariant and satisfies

$$
\begin{aligned}
& H(p)=\int_{X} h(x) d p(x) \quad \text { for every } p \in \boldsymbol{P}_{\mathrm{S}} \\
& h(x)=h_{p}(x) \quad \text { p-a.e. } x \in X \text { and for every } p \in \boldsymbol{P}_{\mathrm{S}} .
\end{aligned}
$$


This function $h(x)$ was introduced by Parthasarathy in case of $X=A^{I}$. The proof will be also done along his construction, combined with Theorems 2 and 3, in which use is made of the well-known theorem of Kryloff-Bogoliouboff relative to the ergodic decomposition of invariant measure, cf. Oxtoby [6]. Before the proof, we shall give a preliminary and several lemmas.

Let $\mathfrak{U}$ be the field, of clopen sets, generated by $\left\{S^{n} U ; U \in \mathscr{Z}, n=0, \pm 1, \pm 2, \cdots\right\}$, and $\mathfrak{B}$ the $\sigma$-field of Borel sets generated by $\mathfrak{H}$. Putting $\boldsymbol{C}_{\mathscr{E}}$ the uniformly closed linear subspace of $C(X)$ generated by $\left\{C_{U} ; U \in \mathfrak{U}\right\}$, then $\boldsymbol{C}_{\mathscr{E}}$ is uniformly separable and has a countable dense subset $\left\{f_{n}\right\}_{k=1}^{\infty} \subset \boldsymbol{C}_{\mathscr{Z}}$. Putting

$$
d(x, y)=\sum_{n=1}^{\infty} \frac{\left|f_{n}(x)-f_{n}(y)\right|}{2^{n}}\left\|f_{n}\right\|, \quad x, y \in X,
$$

$d(\cdot, \cdot)$ is a quasi-metric on $X$, and denote $x \sim y(x, y \in X)$ if and only if $d(x, y)=0$. Moreover, put $\tilde{X}=X / \sim$ the quotient space of $X$ with respect to the equivalence relation $\sim$ and put

$$
\tilde{d}(\widetilde{x}, \widetilde{y})=d(x, y) \quad \text { for each pair } \quad x, y \in X,
$$

where $\tilde{x}(x \in X)$ is the class containing $x \in X$. Then $(\tilde{X}, \tilde{d})$ is a compact metric space, the canonical mapping $x \rightarrow \tilde{x}$ from $X$ onto $\tilde{X}$ is continuous, and $\boldsymbol{C}_{\mathscr{Z}}$ is isomorphic with $C(\tilde{X})$ under the isomorphism $f \in \boldsymbol{C}_{\mathscr{Z}} \rightarrow \tilde{f} \in C(\tilde{X})$ defined by that, for each $x \in X$

$$
f(y)=\tilde{f}(\tilde{x}) \quad \text { for every } y \in \tilde{x} .
$$

Furthermore, $\tilde{\mathfrak{B}}=\{\tilde{B} ; B \in \mathfrak{B}\}(\tilde{B}=\{\tilde{x} \in \tilde{X} ; x \in B\})$ is the $\sigma$-field of all Borel sets in $\tilde{X}$, and hence the function $\widetilde{f}(\tilde{x})$ defined by (9) is Borel measurable on $\tilde{X}$ if and only if $f(x)$ is $\mathfrak{B}$-measurable on $X$. Putting $\widetilde{S}: \widetilde{x} \rightarrow(S x)^{2}$, which is well defined mapping on $X$ onto $\tilde{X}$ because $d(x, y)=0$ if and only if $d(S x, S y)=0$, then $\tilde{S}$ is a homeomorphism on $\tilde{X}$.

LemMA 1. For any non-negative linear functional $\rho$ on $\boldsymbol{C}_{\mathscr{E}}$ with norm one, there corresponds a probability measure $\mu_{\rho}$ over the measurable space $(X, \mathfrak{B})$ such that

$$
\rho(f)=\int_{X} f(x) d \mu_{\rho}(x) \quad \text { for every } \quad f \in C_{\nsubseteq} .
$$

Proof. This follows from the Riesz theorem. Putting $\tilde{\rho}(\widetilde{f})=\rho(f), f \in \boldsymbol{C}_{\mathscr{z}}$, then $\tilde{\boldsymbol{\rho}}$ is a non-negative linear functional on $C(\tilde{X})$ with norm one and hence there exists a regular probabiltiy measure $\mu_{\tilde{\rho}}$ on $\tilde{X}$ such that

$$
\tilde{\rho}(\widetilde{f})=\int \widetilde{f}(\tilde{x}) d \mu_{\tilde{\rho}}(\tilde{x}) \quad \text { for every } \tilde{f} \in C(\tilde{X}) .
$$

Put $\mu_{\rho}(B)=\mu_{\tilde{\rho}}(\tilde{B})$ for every $B \in \mathfrak{B}$, then $\mu_{\rho}(\cdot)$ is a regular probability measure over $(X, \mathfrak{B})$ and $(10)$ follows from (11).

LEMMA 2. If the functional $\rho$ given in Lemma 1 is S-stationary, i.e. $\rho(S f)$ $=\rho(f)((S f)(x)=f(S x))$, then the corresponding measure $\mu_{\rho}$ is S-invariant. 
Indeed, this follows immediately from that

$$
\mu_{\rho}\left(S^{-1} V\right)=\rho\left(S C_{V}\right)=\rho\left(C_{V}\right)=\mu_{\rho}(V) \quad \text { for every } V \in \mathfrak{H} .
$$

Now, we refer, as Parthasarathy [7], the notion of Kyloff-Bogoliouboff's (K-B, say) theorem; cf. Oxtoby [6]. Denote

$$
M_{x},{ }_{n}(f)=\frac{1}{n} \sum_{k=1}^{n} f\left(S^{k} x\right) \quad \text { for each } x \in X \text { and } f \in C_{\mathscr{C}},
$$

$n=1,2, \cdots$. If the limit of $M_{x},{ }_{n}(f)(n \rightarrow \infty)$ exists for every $f \in C_{\mathscr{\not}},=M_{x}(f)$ say, then it is non-negative and $S$-stationary linear functional, with norm one, on $\boldsymbol{C}_{\mathscr{}}$. Such an $x \in X$ is called a quasi-regular point in $X$ relative to $\boldsymbol{C}_{\ddagger}$. Denote $Q$ the set of all such points $x \in X$. Then, by Lemma 2, for each $x \in Q$, there corresponds an $S$ invariant measure $m_{x}=\mu_{M_{x}}$ over $(X, \mathfrak{B})$ such that

$$
M_{x}(f)=\int_{X} f(y) d m_{x}(y) \quad \text { for every } \quad f \in \boldsymbol{C}_{\mathscr{F}},
$$

and $m_{x}$ satisfies

$$
m_{x}(B)=m_{S x}(B) \quad \text { for every } B \in \mathfrak{B} \text { and for every } \quad x \in Q .
$$

If $m_{x}$ is ergodic with respect to $S$ over $(X, \mathfrak{B})$, then $x$ is called a regular pornt in $X$ relative to $\boldsymbol{C}_{\mathscr{z}}$. Denote $R$ the set of all regular points in $X$. Then K-B theorem implies that

$$
Q \in \mathfrak{B}, R \in \mathfrak{B} \text { and } p(Q)=p(R)=1 \quad \text { for every } \quad p \in \boldsymbol{P}_{S} .
$$

Indeed, since $\tilde{X}$ is compact metric space with homeomorphism $\widetilde{S}$, and since for each $x \in X$

$$
M_{x, n}(f)=\frac{1}{n} \sum_{k=1}^{n} \tilde{f}\left(\widetilde{S}^{k} \tilde{x}\right) \quad \text { for every } \tilde{f} \in C(\tilde{X})
$$

and $\tilde{Q}$ or $\tilde{R}$ are the sets of all quasi-regular or regular point in $\tilde{X}$ relative to $C(\tilde{X})$, respectively, and both $\tilde{Q}, \widetilde{R}$ are Borel sets in $\tilde{X}$ and invariant measure one, i.e., $\tilde{p}(\widetilde{Q})=\tilde{p}(\widetilde{R})=1$ for every $p \in \boldsymbol{P}_{\text {s }}$ (cf. Oxtoby [6], (2. 4)), where $\tilde{p}$ is the $\widetilde{S}$-invariant and regular probability measure over $\tilde{X}$ defined by $\tilde{p}(\tilde{B})=p(B), B \in \mathfrak{B}$, that is, (14) holds.

LEMMA 3. For each bounded $\mathfrak{B}$-measurable functions $f$ on $X,^{1)}$

$$
\int_{X} f(x) d m_{r}(x),=f^{\natural}(r) \quad s a y,
$$

1) The mapping $f \rightarrow f^{\natural}$, defined over the Banach space of all bounded $\mathfrak{B}$-measuable functions $B(X)$ (with sup-norm) into itself, concides with the concept of the expectation in the sense of Nakamura-Turumaru [5] and also the conditional expectation in the sense of Umegaki [8]. 
$\left(f^{\natural}(r)=M_{r}(f)\right.$ if $\left.f \in \boldsymbol{C}_{\mathscr{E}}\right)$, is a bounded, B-measurable and S-invariant function over $R$, and it satisfies

$$
\int_{X} f(x) d p(x)=\int_{R} f^{\natural}(r) d p(r)=\int_{R}\left(\int_{X} f(x) d m_{r}(x)\right) d p(r) .
$$

This follows from K-B Theorem (cf. [6], (2. 6)) that, for $r \in R$

$$
f^{\natural}(r)=\int_{\tilde{X}} \tilde{f}(\tilde{x}) d m_{\tilde{r}}(\tilde{x}), \quad=\widetilde{g}(\tilde{r}) \quad \text { say, }
$$

and $\widetilde{g}$ is Borel measurable over $\widetilde{R}$, and that

$$
\int_{\tilde{R}} \tilde{g}(\tilde{r}) d \tilde{p}(\tilde{\boldsymbol{r}})=\int_{\tilde{x}} \tilde{f}(\tilde{x}) d \tilde{p}(\tilde{x})
$$

The S-invariance of $f^{\natural}(x)$ follows from (13).

Proof of Theorem 5. Put $\boldsymbol{P}_{S}^{\prime}$ be the set of all $S$-invariant probability measures over the measurable space $(X, \mathfrak{B})$. Then the theorems and their proofs in $\S 3$ hold for $p, q, \cdots$ in $\boldsymbol{P}^{\prime}$ s without chainging their statements. Since $m_{r} \in \boldsymbol{P}^{\prime}$ s for each $r \in R$, the function $h_{m_{r}}(x)$ over $X$ can be defined by (5). Putting

$$
h(r)=\left\{\begin{array}{lll}
\int_{X} h_{m_{r}}(x) d m_{r}(x) & \text { for } & r \in R, \\
0 & \text { for } r \notin R,
\end{array}\right.
$$

(i.e., $h(r)=H\left(m_{r}\right)$ for $r \in R$ ), then $h(\cdot)$ is bounded, $S$-invariant and $\mathfrak{B}$-measurable. Indeed, put $h_{n}(r, x)=h_{m_{r}},{ }_{n}(x)(r \in R)$ and put

$$
g_{n}(r)=\int_{X} h_{n}(r, x) d m_{r}(x)=-\sum_{U \in \mathscr{E}} \sum_{V \in \mathscr{E}_{n}} m_{r}(U \frown V)\left[\log m_{r}(U \frown V)-\log m_{r}(V)\right]
$$

Whence, since $m_{r}(W)\left(=M_{r}\left(C_{W}\right), W \in \mathfrak{H}\right)$ is $\mathfrak{B}$-measurable on $R$, so is $g_{n}(r)$ on $R$. Furthermore, as in the proof of Theorem 3 , since $h_{n}(r, x) \downarrow h_{m_{r}}(x),=h(r, x)$ say,

$$
h(r)=\int_{X} h(r, x) d m_{r}(x)=\lim _{n \rightarrow \infty} g_{n}(r) \quad(r \in R)
$$

and $h(r)$ is $\mathfrak{B}$-measurable on $R$ and hence $h(x)$ is so on $X$. The boundedness of $h(x)$ follows from (5) for $p=m_{r}$ and the definition (17) of $h(x)$, and S-invariance follows from $m_{r}=m_{\mathrm{S} r}$.

As Parthasarathy ([7], Theorem 2. 6), for each $U \in \mathscr{L}, V \in \mathscr{F}_{\infty}$ and $p \in \boldsymbol{P}_{\mathrm{S}}$, and for every fixed $q \in \boldsymbol{P}_{\mathrm{S}}, q \ll p$,

$$
q(U \frown V)=\int_{V} P_{q}\left(U \mid \mathscr{I}_{\infty}\right)(x) d q(x)=\int_{V} P_{p}\left(U \mid \mathscr{\not}_{\infty}\right)(x) d q(x) \quad \text { (by Theorem 2) }
$$




$$
=\int_{R}\left(\int_{V} P_{p}\left(U \mid \mathscr{F}_{\infty}\right)(x) d m_{r}(x)\right) d q(r) \quad \text { (by Lemma 3) }
$$

and

$$
\begin{array}{rlrl}
q(U \frown V) & =\int_{X} C_{U \sim V}(x) d q(x)=\int_{R}\left(C_{U \sim V}\right)^{\natural}(r) d q(r)=\int_{R} m_{r}(U \frown V) d q(r) \\
& =\int_{R}\left(\int_{V} P_{m_{r}}\left(U \mid \mathscr{L}_{\infty}\right)(x) d m_{r}(x)\right) d q(r) & \text { (by Lemma 3). }
\end{array}
$$

This and (13) imply that $P_{p}\left(U \mid \mathscr{F}_{\infty}\right)(x)=P_{m_{r}}\left(U \mid \mathscr{F}_{\omega}\right)(x)$ and

$$
h_{p}(x)=h_{m_{r}}(x) \text { for } m_{r} \text {-a.e. } x \in X \text { and for each fixed } r \in R
$$

within p-a.e. $r$ in $R$. Thus we obtain the required equality (7):

$$
\begin{aligned}
H(p) & =\int_{X} h_{p}(x) d p(x)=\int_{R} h_{p}^{\natural}(r) d p(r)=\int_{R}\left(\int_{X} h_{p}(x) d m_{r}(x)\right) d p(r) \\
& =\int_{R}\left(\int_{X} h_{m_{r}}(x) d m_{r}(x)\right) d p(r)=\int_{R} h(r) d p(r)=\int_{X} h(x) d p(x) .
\end{aligned}
$$

Besides, by Theorems 2, 3 and (7), whenever $q \ll p\left(p, q \in \boldsymbol{P}_{s}\right)$,

$$
H(q)=\int_{X} h_{q}(x) d q(x)=\int_{X} h_{p}(x) d q(x)=\int_{X} h(x) d q(x)
$$

hence

$$
\int_{X} h(x) f(x) d p(x)=\int_{X} h_{p}(x) f(x) d p(x)
$$

for every $f \in L^{1}(X, p)$, S-invariant. Since both $h$ and $h_{p}$ are $S$-invariant, (8) is obtained.

The function $h(x)$ will be called universal entropy function associated with the clopen partition $\mathscr{F}$ and the homeomorphism $S$, and sometimes precisely denote $h(x)=h(x, \mathcal{F}, S)$. Let $\boldsymbol{L}$ be the Banach space of all bounded signed regular measures over $X$ with the norm of total variation. Then putting

$$
H(\xi)=\int_{X} H(x) d \xi(x) \quad \text { for every } \quad \xi \in \boldsymbol{L},
$$

$H(\cdot)$ is a bounded non-negative definite linear functional over $\boldsymbol{L}$, and is $S$-stationary, i.e., $H(S \xi)=H(\xi)$ for every $\xi \in \boldsymbol{L}$, where $S \xi \in \boldsymbol{L}$ is defined by $(S \xi)(V)=\xi\left(S^{-1} V\right)$ for every Borel set $V$ in $X$. The functional $H(\xi)$ over $\boldsymbol{L}$ coincides with the functional 
$H(\xi, \mathscr{Z}, S)$ in the paper [9],2) and again it is called the entropy functional over $\boldsymbol{L}$ associated with a clopen patition $\not{z}$ and a homeomorphism $S$.

It is the author's pleasure to acknowledge that he gets valuable discussions from Prof. M. Nakamura.

\section{REFERENCES}

[1] BReiman, L., On achieving channel capacity in finite-memory channels. Ill. J. Math. 4 (1960), 246-252.

[2] Dixmier, J., Sur certains especes considérés par M. H. Stone. Sum. Bras. Math. 11 (1951), 1-32.

13] Dixmier, J., Les algèbres d'opérateurs dans l'espace Hibertien. Paris (1957).

[4] Halmos, P. R., Entropy in ergodic theory, Univerity of Chicago (1959).

[5] Nakamura, M., and T. Turumaru, Expectations in an operator algebra. Tôhoku Math. J. 6 (1954), 189-204.

[6] Oxтовy, J. C., Ergodic sets. Bull. Amer. Math. Soc. 58 (1952), 116-136.

[7] Parthasarathy. K. R., On the integral representation of the rate of transmission of a stationary channel. Ill. J. Math. 5 (1961), 299-305.

[8] Umegaki, H., Conditional expectation in an operator algebra. Tôhoku Math. J. 6 (1954), 177-181.

[9] Umegaki, H., A functional method on amount of entropy. Kōdai Math. Sem. Rep. 15 (1963), 162-175.

Department of Mathematics,

Tokyo Institute of Technology.

2) In the preceding paper [9], $\S 4$, it was assumed that if the measurable space $(X, \mathfrak{X})$ with measurable transformation $S$ has denombrable generator, then it has maximal $S$-invariant probabitity measure relative to the ordering « of absolute continuity. However, in general, this does not hold, for example, when $S$ is identity mapping from $X$ onto $X$, $X=$ the interval $[0,1]$ and $\mathfrak{X}$ is $\sigma$-field of Borel subsets. Therefore, it should be corrected such as

P. 168, line 3 line 4 "Then $P(X, S)$ is necessarily...dominates all $p \in P(X, S)$ " reads such as

"Assume that there exists an S-invariant probability measure $\mu$, and denote $P(X, S)$ (resp., $L(X, S)$ ) the sets of all $S$-invariant 'probability' (resp., 'bounded signed') measures $p, \cdots$ (resp., $\xi, \cdots$ ) which are absolutely continuous with respect to $\mu . "$

Hence in the parts below in [9] the dominatedness for the sets $P(X, S)$ and $L(X, S)$ of measures should be assumed.

However, the measurable space $(X, \mathfrak{X})$ given in the paper [9], can be represented by a totally disconnected compact space in preserving the measurability structure and where the measurable transformation $S$ is mapped to a homeomorphism. Therefore, by Theorem 5 and the discussions in $\S 4$ of the present paper, the theorems in [9] hold without assuming the denombrability of the measurable space. 